\title{
Ist der „mündige Verbraucher“ eine Fiktion?* \\ Ein kritischer Beitrag zum aktuellen Stand der Diskussion um das Verbraucherleitbild in den Wirtschaftswissenschaften und der Wirtschaftspolitik
}

\section{PETER KENNINg UND INGA WOBKER ${ }^{* *}$}

$\mathrm{Da}$ ein einheitliches, politikfeldübergreifendes und wirtschaftswissenschaftlich fundiertes Verbraucherleitbild fehlt, gibt dieser Beitrag einen Überblick über den Stand der wirtschaftswissenschaftlichen Diskussion um das Verbraucherleitbild. Eine empirische Analyse zeigt, dass es „den mündigen Verbraucher" nicht gibt und eine differenzierte Betrachtung nötig ist. Am Beispiel des Verbraucherbegriffs des Gesetzes gegen Wettbewerbsbeschränkungen werden Implikationen für die Wirtschaftspolitik diskutiert.

Schlagwörter: Verbraucherleitbild, Verbraucherforschung, Verbraucherpolitik, Wettbewerbspolitik, Verhaltensökonomik

\section{A Critical Contribution to the Current State of the Debate on the Consumer Model in Economics and Economic Policy}

In Germany there is still no uniform, policy field-spanning and economic research-based consumer model. This paper provides an overview of the contributions of economic research to the discussion about the consumer model. Scrutinizing this model with an empirical analysis, we find that the picture of "the empowered consumer" does not exist, and rather a more differentiating approach would be

Beitrag eingereicht am 16.11.2012; nach doppelt verdecktem Gutachterverfahren überarbeitete Fassung angenommen am 11.07.2013.

Univ-Prof. Dr. Peter Kenning, Lehrstuhl für Marketing, Zeppelin Universität, Am Seemooser Hern 20, D-88045 Friedrichshafen, Tel.: +49-(0)7541-6009-1271, Fax: +49-(0)7541-6009-1299, E-Mail: peter.kenning@zu.de, Forschungsschwerpunkte: Konsumentenverhalten, Verbraucherforschung, Consumer Neuroscience, Neuroökonomik, Handelsmarketing.

Inga Wobker (M.A.), Lehrstuhl für Marketing, Zeppelin Universität, Am Seemooser Hern 20, D-88045 Friedrichshafen, Tel.: +49-(0)7541-6009-1275, Fax: +49-(0)7541-6009-1299, E-Mail: i.wobker@zeppelin-university.net, Forschungsschwerpunkte: Konsumentenverhalten, Verbraucherforschung, Emotionen, Behavioral Economics.

Die folgenden Ausführungen sind zum Teil das Ergebnis einer umfassenden Diskussion des Erstautors mit verschiedenen verbraucherpolitisch aktiven Wissenschaftlern, denen wir zu Dank verpflichtet sind. Er gilt den Mitgliedern des wissenschaftlichen Beirats „Verbraucher- und Ernährungspolitik“ beim Bundesministerium für Ernährung, Landwirtschaft und Verbraucherschutz (BMELV) und seinem Vorsitzenden Andreas Oehler (Universität Bamberg), Lucia Reisch (Copenhagen Business School) sowie den TeilnehmerInnen des ersten Fachforums des Netzwerks Verbraucherforschung zum Thema „Alternativen zum Informationsparadigma der Verbraucherpolitik" im Januar 2013 an der Zeppelin Universität in Friedrichshafen. Die Ausführungen zum Thema „Wettbewerbs- und Kartellrecht“ wurden maßgeblich beeinflusst durch Diskussionen und Gespräche mit Dieter Ahlert (Universität Münster), Hendrik Schröder (Universität Duisburg-Essen) sowie Rainer Olbrich und Gundula Grewe (beide Fernuniversität Hagen). 
necessary. Using the consumer concept of the Law against Restraints on Competition, implications of this evidence-based differentiation for economic policy are discussed.

Keywords: Consumer Model, Consumer Research, Consumer Policy, Competition Policy, Behavioral Economics

\section{Einleitung}

In den letzten etwa zwanzig Jahren hat es in der wirtschaftswissenschaftlichen Forschung und wirtschaftlichen Praxis eine erstaunliche Bedeutungszunahme verhaltenswissenschaftlicher Ansätze gegeben (vgl. Oehler/Reisch 2008). Diese Bedeutungszunahme ist nicht ohne politische Wirkung geblieben. Vielmehr hat sich die Wirtschaftspolitik parteiübergreifend dieser Ansätze bedient und orientiert sich immer stärker an den realen, empirischen Gegebenheiten und dem beobachteten Verbraucherverhalten als an theoretischen Modellen. Im Zuge dieser zunehmenden empirischen, verhaltenswissenschaftlichen Ausrichtung, deren Bedeutung von den politischen Parteien jedoch noch sehr unterschiedlich gesehen wird, entstanden in verschiedenen wirtschaftspolitisch relevanten Politikbereichen neue Institutionen und Instrumente. ${ }^{1}$ Damit einher ging jedoch ein oft noch ungeklärtes Verhältnis zum Begriff des „souveränen“ oder auch „mündigen Verbrauchers“ (vgl. Srnka/Schweitzer 2000). Dies gilt für verschiedene Bereiche der Wirtschaftspolitik, wie z.B. die Wettbewerbs- sowie auch und insbesondere für die Verbraucherpolitik (vgl. BMELV 2012). Somit bleibt bis dato ungeklärt, welches Verbraucherleitbild den politischen Aktionen deskriptiv zugrunde liegt bzw. mit den entsprechenden Maßnahmen auf einer normativen Ebene eigentlich angestrebt werden sollte (vgl. z.B. Micklitz et al. 2010). Zudem bleibt in Ermangelung einer interdisziplinär angelegten Verbraucherforschung bzw. wissenschaft unklar, welche Domäne (z.B. Soziologie, Psychologie, Jura, Wirtschafts-, Politik- oder Neurowissenschaften) die für die Definition notwendige Legitimation für sich beanspruchen darf. Es verwundert daher nicht, dass es derzeit kein einheitliches, politikfeldübergreifendes Verbraucherleitbild gibt. Stattdessen wird „das“ Leitbild oft von verschiedenen Interessensgruppen instrumentalisiert (u.a. Strünck et al. 2010). Gleichwohl spielt gerade im rechtlichen Bereich die Orientierung an einem deskriptiven bzw. normativen Leitbild eine wichtige Rolle, wie bspw. an den aktuellen Entwicklungen auf europäischer Ebene deutlich wird. Vermutlich auch deswegen wurde auf dem Juristentag 2012 in München der Ruf laut, zivilrechtliche Entscheidungen künftig differenzierter zu treffen, als dies bei der Verwendung eines einheitlichen Leitbilds in der Vergangenheit oft der Fall gewesen ist.

Vor diesem Hintergrund soll der vorliegende Beitrag den aktuellen Diskussionstand um „das" Verbraucherleitbild bzw. „den“ mündigen Verbraucher aus einer wirtschaftswissenschaftlichen Perspektive beleuchten. Dazu werden zunächst die verschiedenen Paradigmen der ökonomischen Forschung hinsichtlich ihrer Diskussions-

1 Man denke hier beispielsweise an das im Jahre 2012 vom BMELV erstmalig vorgelegte „Gutachten zur Lage der Verbraucherinnen und Verbraucher in Deutschland" sowie die Initiativen im von der Bundesanstalt für Landwirtschaft und Ernährung (BLE) organisierten Netzwerk Verbraucherforschung (vgl. Grugel 2012). 
relevanz gewürdigt. Im Ergebnis zeigt sich ein Zustand, den man als informationsökonomischen Flickenteppich bezeichnen könnte und der angesichts aktueller wissenschaftlicher Erkenntnisse, insbesondere aus dem Bereich der Verhaltens- und Neuroökonomik (vgl. Priddat 2007) wohl dringend einer Weiterentwicklung bedarf. Da diese Weiterentwicklung nicht ohne empirische Fundierung im Sinne einer bereits von verbraucherwissenschaftlicher Seite vielfach geforderten Evidenzbasierung auskommen wird, werden im dritten Kapitel die Ergebnisse einer empirischen Studie zum ökonomischen Wissen der Verbraucher auszugsweise vorgestellt. Im Ergebnis zeigt sich, dass es „den“ Verbraucher nicht gibt, sondern vielmehr verschiedene Typen situationsspezifisch zu unterscheiden und durch unterschiedliche Problemlösungsfähigkeiten gekennzeichnet sind. Da diese Differenzierung für den Verbraucherbegriff in verschiedenen wirtschaftspolitischen Bereichen von Bedeutung sein dürfte, wird im vierten Kapital exemplarisch an dem Gesetz gegen Wettbewerbsbeschränkungen (GWB) gezeigt, welche Gefahren mit einer undifferenzierten Betrachtung einhergehen. Abschließend wird ein kurzes Fazit gezogen und ein Ausblick gegeben.

\section{Das Verbraucherleitbild in den Wirtschaftswissenschaften - ein informa- tionsökonomisch eingefärbter Flickenteppich?}

In den wirtschaftswissenschaftlichen Theorien ist es verbreitet, die Verbraucher als weitgehend autonome Individuen zu sehen, die selbstbestimmt handeln können, wollen und sollen (vgl. hierzu und zum Folgenden Strünck et al. 2012) und somit in der Lage sind, das Marktangebot „souverän“ zu steuern (vgl. Srnka/Schweitzer 2000: 193). In einer weiterführenden Analyse der verschiedenen theorieinhärenten Modelle können zunächst grob zwei Modellarten unterschieden werden: Steht bei den normativen Modellen die Frage im Vordergrund, wie Menschen sich - aus welchen normativen Gründen auch immer - verhalten sollten, geht es bei den deskriptiven Modellen hingegen darum, zu beschreiben, wie Menschen sich tatsächlich, d.h. in der Realität, verhalten. Da die deskriptiven Modelle oft die Basis für die Ableitung normativer Modelle darstellen, sollen im Folgenden zunächst die deskriptiven Modelle gewürdigt werden. Diese Modelle sind nach dem Kriterium der mit der wissenschaftlichen Weiterentwicklung verbundenen zeitlichen Entwicklung in verschiedene Entwicklungsstufen unterteilbar (vgl. Abb. 1). Heute kann man eine Koexistenz der Modelle beobachten, so dass es derzeit kein einheitliches Modell gibt. Gemeinsam ist ihnen aber, dass sie oft durch besonders rigide und realitätsferne Annahmen gekennzeichnet sind. Beispielsweise wird in den nach wie vor populären neoklassischen Modellen unterstellt, dass Verbrauchern sämtliche Informationen frei zugänglich wären, sie diese eindeutig verstehen, verarbeiten, erinnern und abrufen würden. Außerdem gehen diese Modelltypen davon aus, dass die Präferenzen der Verbraucher „starr“ und „transitiv“, d.h. widerspruchsfrei sind. Zudem sagen diese Modelle nichts darüber aus, wie diese Präferenzen entstanden sind und beeinflusst werden können; sie sind ex ante ,gegeben“.

Die auf diesen Annahmen basierenden neoklassischen Modelle dienen dann auf der deskriptiven Ebene häufig dazu, allgemeine Aussagen über bestimmte (makro-)ökonomische Sachverhalte treffen zu können, zum Beispiel zur Entwicklung von Märkten oder zum Verhalten der Marktteilnehmer. Auch ist mit diesen Modellen eine für die Wirtschaftspolitik wichtige Beschreibung der Verbraucher nach dem Leitbild der Konsumentensouveränität möglich (vgl. Srnka/Schweitzer 2000: 193). Die mit 
den Annahmen verbundene Realitätsferne sowie das Versagen der Modelle, individuelles Verbraucherverhalten hinreichend erklären zu können, werden von der Vertretern dieses Modelltyps zum Teil bewusst in Kauf genommen („as-if-Ansatz"). Der zentrale Grund hierfür besteht wohl darin, die Modelle mathematisch beherrschbar zu halten. Zum Teil wird aber auch behauptet, der effiziente Markt würde nichttheoriekonformes Verhalten von selbst beheben (vgl. Fama 1970). Es ist wohl evident, dass dieser Modellplatonismus in der Realität überaus problematisch und wenig nützlich ist (vgl. z.B. Frey 1990). Denn tatsächlich versagen die neoklassischen Modelle nahezu regelmäßig, wenn es darum geht, das beobachtbare Verhalten der Verbraucher hinreichend zu erklären.

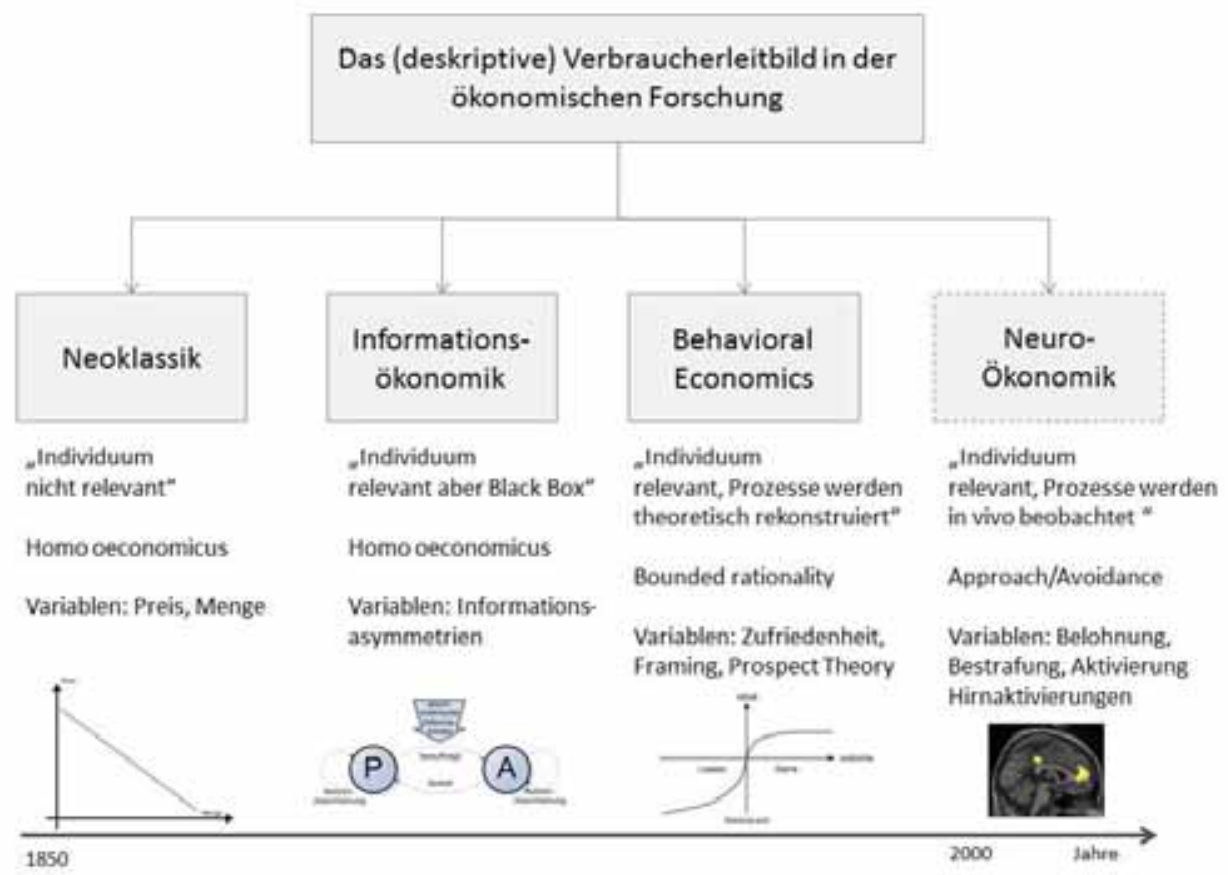

Abbildung 1: Entwicklung alternativer Paradigmen der wirtschaftswissenschaftlichen Verbraucherverhaltensforschung (Quelle: eigene Darstellung)

Dieses Versagen hat in den Wirtschaftswissenschaften zur Entwicklung und Integration neuer Modelle geführt. Ein gerade in der ökonomischen Verbraucherforschung prominentes Modell ist das so genannte „Informationsmodell“ (vgl. z.B. Fritsch et al. 1998). Sein wesentliches Merkmal besteht darin, dass es versucht, zu berücksichtigen, dass zwischen Verbrauchern und Anbietern regelmäßig systematische Informationsasymmetrien bestehen. Im Informationsmodell können schlecht informierte Verbraucher nur beschränkt rational handeln, eben weil sie nicht über die für die richtigen Entscheidungen notwendigen Informationen verfügen (vgl. Strünck et al. 2012). In 
dieser „beschränkten Rationalität“ besteht ein wesentlicher Unterschied zu den neoklassischen Modellen (vgl. z.B. Weiber/Adler 1995a, 1995b). Zudem geht das Informationsmodell davon aus, dass den Verbrauchern nicht alle Informationen unmittelbar sowie kostenlos zur Verfügung stehen und verständlich sind. Die Annahme ist aber, dass, wenn die Verbraucherinnen und Verbrauchern diese Informationen bekämen, sie eigenständig und rational entscheiden würden. Oder, wie es Strünck (2011: 3) formuliert: „Wenn die Informationslücken erst gestopft sind (...), können die Konsumenten zu ihrem eigenen und gesamtwirtschaftlichem Wohl ihre Entscheidungen treffen“. Der Verbraucher ist also auch im Informationsmodell grundsätzlich „mündig“ und „souverän“, er wird daran aber durch fehlende, teure oder unverständliche Informationen gehindert.

Die aus diesem Modell abgeleitete politische Norm ist, dass dem Verbraucher alle relevanten Informationen zugänglich gemacht werden müssen; erst dann ist er hinreichend aufgeklärt, kann „mündig“" werden und „souverän“ entscheiden (vgl. Strünck et al. 2012). Vermutlich auch aufgrund der scheinbar einfachen Umsetzung dieser Norm, übernahmen in der Vergangenheit die Programme fast aller Parteien und auch die Leitsätze der meisten Verbände und Verbraucherorganisationen die Annahmen und Schlussfolgerungen des Informationsmodells. ${ }^{2}$

Im Gegensatz zu dieser Entwicklung wird das Informationsmodell in den Wirtschaftswissenschaften jedoch zunehmend kritisiert. In vorderster Linie erfolgt diese Kritik von Verhaltensökonomen (vgl. z.B. Oehler/Reisch 2008; Kenning/Reisch 2013). Diese Forschungsrichtung arbeitet im Unterschied zur Neoklassik und Informationsökonomik weniger modelltheoretisch, sondern vielmehr empirisch. Ausgangspunkt der Forschungen ist zumeist das durch bestimmte empirische Methoden (z.B. ethnografische Studien, Panels, Experimente) beobachtbare, oft individuelle Verhalten der Verbraucher. Im Gegensatz zur Neoklassik bzw. zum Informationsmodell bildet aber nicht die Theorie, quasi deduktiv, den Ausgangspunkt zur Ableitung des deskriptiven Modells, sondern das reale Verhalten der Verbraucher. Die empirischen Befunde werden zwar im Idealfall induktiv in ein formales Modell überführt und theoretisiert (vgl. Gigerenzer 1991); oft stehen die Befunde aber auch noch ohne theoretischen Unterbau, quasi als Patchwork, mehr oder weniger isoliert nebeneinander. Dennoch zeigen die mittlerweile zahlreichen Befunde ein einheitliches Muster, nämlich, dass es zur Erreichung politischer Ziele nicht ausreicht, fehlende oder falsche Information einfach durch mehr oder bessere Informationen zu ersetzen, da die affektiven und kognitiven Fähigkeiten der Verbraucher kontextspezifisch limitiert sind (vgl. etwa Kahneman 2002; Ariely et al. 2010). In diesem Zusammenhang wurde auch immer deutlicher, dass Informationen bisweilen sogar negative Effekte für das Verbraucherverhalten haben können, u.a. dann, wenn Kundenverwirrung entsteht (vgl.

2 Besonders deutlich wird dies auch im aktuellen Parteiprogramm der FDP: „Wir wollen dem mündigen Verbraucher die notwendigen Informationen für eine freie und fundierte Entscheidung für Einkauf und Ernährung zur Verfügung stellen. Deshalb möchten wir eine konsequente Prozesskennzeichnung für alle Lebensmittel und Konsumgüter, bei deren Produktion an irgendeiner Herstellungsstufe gentechnisch veränderte Organismen beteiligt sind. Nur so ist eine vollständige Aufklärung des Verbrauchers möglich“ (FDP 2013: 24). 
Scheibehenne et al. 2010). Mehr Informationen sind also keineswegs der Königsweg, um Informationsasymmetrien zwischen Anbietern und Nachfragern zu verringern (vgl. Strünck 2011). Demzufolge geht die Verhaltensökonomik nicht nur nicht (sic!) vom Leitbild des mündigen Verbrauchers aus, sondern sie erkennt den mündigen Verbraucher darüber hinaus sogar als unerreichbares Ideal - als wissenschaftliche und politische Fiktion oder „Mythos“ (Strünck et al. 2012: 1).

Im Kern hat die Verhaltensökonomik gezeigt, dass Verbraucherentscheidungen limitiert sind und maßgeblich von drei Faktoren abhängen, nämlich von wem die Informationen geliefert werden, wie diese Informationen präsentiert werden und an welchen Richtwerten sich die Verbraucher orientieren (vgl. Strünck 2011: 3; sowie exemplarisch Deppe et al. 2007). Dabei können die entsprechenden, empirisch gestützten (Teil-)Modelle zwei unterschiedliche Ansatzpunkte verfolgen:

- Primär subjektorientierte Ansätze, die im Wesentlichen individualpsychologische Faktoren, also das Subjekt, als entscheidend für das Verhalten von Verbrauchern ansehen (vgl. grundlegend Gigerenzer 1991; Frey 1990). Zu diesen Ansätzen zählen auch Forschungsarbeiten, die vererbtes - biologisch geprägtes - Verhalten in den Blick nehmen und derzeit unter dem Rubrum der "Consumer Neuroscience" das Verbraucherverhalten erforschen (vgl. z.B. Camerer et al. 2005; Priddat 2007; Kenning et al. 2007; sowie Kenning/Linzmajer 2011). Im Zentrum steht dabei die Annahme, dass biologische Prozesse das Verbraucherverhalten limitieren und der Verbraucher deswegen nicht in der Lage ist, sämtliche zur Verfügung stehende Informationen zu verarbeiten.

- $\quad$ Primär objektorientierte, situative Ansätze, die insbesondere soziale bzw. sozialpsychologische Einflüsse als determinierend diskutieren (z.B. Gruppen-, Rollenmodelle). Hierzu zählen beispielsweise auch Ansätze wie die Haushaltsökonomik (vgl. Piorkowski 2004) oder soziologisch geprägte Ansätze zu verbraucherpolitisch relevanten Phänomenen (z.B. Markenkonsum, vgl. Hellmann 2003). Diese Ansätze verstehen das Verhalten von Verbrauchern primär als durch gesellschaftliche Aspekte bestimmt. In diesem Zusammenhang werden dann auch kollektive Begriffe wie „Milieus“ oder „Community“ verwendet. Diesen Ansätzen zufolge limitieren mithin gesellschaftliche Aspekte das Verbraucherverhalten.

Versteht man das Verbraucherverhalten integrativ als Resultat des Zusammenwirkens subjektiver, objektiver und situativer Faktoren, können auch neuere Phänomene erklärt werden, wie etwa der so genannte „politische Konsum“ (vgl. Schneider 2010): Engagierte Verbraucherinnen und Verbraucher mit hohen sozialen Präferenzen sowie Organisationen und Bewegungen verwenden die sozialen, ökologischen, ökonomischen und politischen Konsequenzen von Konsum als Richtschnur individuellen und politischen Handelns (vgl. Schrader/Diehl 2010).

Zusammenfassend lässt sich festhalten, dass verhaltensökonomische Ansätze die ökonomischen Modelle zwar näher an die (beobachtbare) Realität der Verbraucher heranbringen. Sie anerkennen die begründbare Begrenztheit menschlicher Fähigkeiten und zeigen, dass das Informationsparadigma der Verbraucherpolitik an seine Grenzen gestoßen ist (vgl. Kenning/Reisch 2013). Da parallel dazu aber auch die Bedeutung situativer Faktoren („Lebenslagen“) berücksichtigt werden muss, sinkt gleichzeitig das 
Abstraktionsniveau und die Generalisierbarkeit der (Teil-)Modelle und Aussagen. Ein Umstand, der die Ableitung und Begründung politischer Maßnahmen erheblich erschweren kann und „empirische Entscheidungshilfen“ (Strünck 2011: 165) erfordert, die aber oft noch fehlen. An dieser Stelle wird das „Dilemma der Wirtschaftswissenschaften" (vgl. Strünck et al. 2012: 9) in der Diskussion um ein Verbraucherleitbild erkennbar: Auf der einen Seite gibt es politisch einfache Modelle, die aber auf realitätsfernen Annahmen über das Verhalten und die Fähigkeiten der Verbraucher basieren und daher viel zu grob sind, um die allägliche Realität der Verbraucher ausreichend zu erfassen. Auf der anderen Seite gibt es realitätsnähere Ansätze, die aber politisch sperrig und kleinteilig sind und empirische Entscheidungshilfen benötigen, die in den Verbraucherwissenschaften bis dato nicht entwickelt wurden.

Eine Möglichkeit, dieses Dilemma zu lösen, besteht darin, die zentrale Schwäche der Verhaltensökonomik, nämlich das Fehlen einer einheitlichen theoretischen Basis, durch weitere Forschungen auf aggregierter Ebene zu beheben. Da dies zum jetzigen Zeitpunkt nur durch ein induktiv-exploratives Vorgehen gelingen kann, wird in diesem Forschungsfeld sehr datenintensiv, gleichwohl aber noch unsystematisch, gearbeitet. Im verbraucherpolitischen Kontext wird somit oft die Forderung zur Einrichtung entsprechender Datenpools oder -sammelstellen im Sinne einer evidenzbasierten Methodik erhoben (vgl. Hagen 2011; Hagen et al. 2013; Strünck et al. 2012: 13). Mit anderen Worten: Die entsprechenden verhaltensökonomischen Instrumente müssen ihre Validität künftig in der breiteren gesellschaftlichen Praxis nachweisen und das Labor verlassen. Dazu erscheint es aber unabdingbar, ihre Wirkung in der gesellschaftlichen Praxis mit Hilfe geeigneter Methoden und möglichst systematisch zu beobachten und politisch brauchbare, empirisch fundierte Entscheidungshilfen zu entwickeln. Vor diesem Hintergrund erklären sich auch die aktuellen verbraucherpolitischen Aktivitäten, wie bspw. die Etablierung des Netzwerks Verbraucherforschung seitens der BLE sowie die erstmalige Vergabe eines Gutachtens zur Lage der Verbraucherinnen und Verbraucher in Deutschland seitens des BMELV (vgl. Grugel 2012).

Neben den bis hierhin geschilderten deskriptiven Ansätzen, die primär darauf abzielen, das Verhalten der Verbraucher (besser) zu beschreiben, zeigt die wirtschaftswissenschaftliche Leitbilddiskussion seit etwa zehn Jahren aber auch zunehmend normative Züge (vgl. z.B. Srnka/Schweitzer 2000). Besonders deutlich wird dies an der wachsenden Diskussion um den Begriff des „nachhaltigen Konsums“ oder seinem Vorläufer, dem ökologisch verantwortlichen Kaufverhalten. In dieser Diskussion steht u.a. die Frage im Raum, ob und, wenn ja, wie die möglichen negativen Folgen von Konsum für die individuellen Entscheidungen der Verbraucher eine Rolle spielen bzw. spielen sollten. Das wohl zentrale Merkmal dieser Diskussion ist das der (intergenerativen) Verteilungsgerechtigkeit (vgl. Schrader und Diehl 2010).

Durch dieses Merkmal geht die ethisch-normative Modellierung - die man auch als „verantwortungsvollen Konsum“ bezeichnen könnte - über die rein deskriptive Modellierung individuellen Verhaltens hinaus. Vielmehr werden die Verbraucher aufgefordert, Verantwortung für ihren Konsum zu übernehmen (vgl. Srnka und Schweitzer 2000). Da der Konsum die Verteilung ökonomischer Chancen zwischen heutigen Generationen ebenso wie die Chancen zwischen heutigen und zukünftigen Generationen beeinflusst, sollte ein entsprechend ethisch-normativ geprägtes Verbraucherleit- 
bild eine auch zeitlich weiter gefasste Berücksichtigung von Interessen aufweisen. Inwiefern Kriterien der (intergenerativen) „Verteilungsgerechtigkeit" in individuelle Entscheidungen einfließen können und müssen, soll im Modell des „verantwortungsvollen Konsumenten" analysiert werden. Mit diesem kollektiven und intergenerativen Anspruch geht das Modell des nachhaltigen Konsums in seinen Annahmen und Forderungen deutlich über die bisher genannten, deskriptiven Modelle der Neoklassik, Informations- und Verhaltensökonomik hinaus. Gleichzeitig bewegt es sich aber auch auf einer anderen Ebene als die praktisch-normativen Modelle der wirtschaftswissenschaftlichen Theorie, indem es dem Modell des mündigen, weil nutzenmaximierenden Verbrauchers eine neue Facette hinzufügt: die der gesellschaftlichen Verantwortung. Dabei wird implizit unterstellt, dass die Verbraucher in der Lage sind, mündig oder „souverän“ zu entscheiden, und bspw. zu erkennen, mit welchen Konsumentscheidungen sie ihrer neuen, verantwortungsvollen Rolle nachkommen können und welche Entscheidungen eher verantwortungslos sind. Im Kern basiert das Konzept des verantwortungsvollen Konsums somit ebenfalls auf der nicht zutreffenden Annahme, es gebe „den“ mündigen Verbraucher bereits heute schon. Auf der ethisch-normativen Ebene ist der mündige Verbraucher somit das Ideal; er ist aber ebenfalls nicht real.

Als Zwischenfazit lässt sich also festhalten, dass das Leitbild des mündigen Verbrauchers in der ökonomischen Forschung mehrere deskriptive und normative Funktionen und verschiedene Phasen durchlaufen hat (vgl. zusammenfassend Abb. 1). In der Neoklassik wird seine Existenz postuliert, um die Modelle beherrschbar zu halten. Dort ist er nicht mehr als eine weitgehend ungeprüfte, gegebene Modellannahme. In der Informationsökonomik wird erkannt, dass diese Annahme nur dann zutreffend ist, wenn bestimmte Voraussetzungen erfüllt sind und Informationsasymmetrien überwunden werden können. Die Verhaltensökonomik hingegen bezweifelt seine gegenwärtige und zukünftige Existenz grundsätzlich. In ihrer jüngsten Form, der Neuroökonomik, erforscht sie die damit verbundenen (neuro-)biologischen Faktoren. Und in der ebenfalls noch jungen, eher ethisch-normativ geprägten Diskussion um nachhaltigen bzw. verantwortungsvollen Konsum formulieren die Wirtschaftswissenschaften schließlich die Anforderungen neu, die gelten sollten, damit der Verbraucher „mündig“ ist, um gleichzeitig zu betonen, dass dieses Verhalten gesellschaftlich notwendig und ressourcenorientiert erforderlich ist.

Aus den bisherigen Ausführungen wurde ersichtlich, dass es "das“ einheitliche Verbraucherleitbild und „den“ mündigen Verbraucher aus Sicht der wirtschaftswissenschaftlichen Forschung offenkundig nicht gibt bzw. nicht geben kann. Vielmehr scheint es angesichts der verhaltensökonomischen Befunde geboten, eine differenzierte, den jeweiligen Situationen („Lebenslagen“) angemessene, empirisch informierte Politik zu verfolgen (vgl. Strünck et al. 2012: 13). Um einen ersten Schritt in Richtung Differenzierung zu gehen, wurde in der verbraucherpolitischen Diskussion unlängst eine Dreiteilung in drei verschiedene Verbrauchertypen diskutiert, nämlich den verantwortungsvollen, den vertrauenden und den verletzlichen Verbraucher (vgl. hierzu und zum Folgenden: Micklitz et al. 2010). ${ }^{3}$ Auch wenn diese Differenzierung in vielen

3 Diese im Deutschen etwas ungenau und ungelenk klingenden Begriffe sind wörtliche Übersetzungen aus dem Englischen. In der Forschung spricht man z.B. vom „,confident consumer“ (ver- 
Situationen zu grob erscheint, kann man diese drei Typen im Allgemeinen durch die folgenden Merkmale kennzeichnen.

Merkmal der verletalichen Verbraucher ist ihre relativ geringe Problemlösungsfähigkeit, die oft auch mit fehlenden Kenntnissen über Produkte, Angebote und Rechte einhergeht. Die verletzlichen Verbraucherinnen und Verbraucher entsprechen am wenigsten dem Leitbild des „mündigen“ Verbrauchers und stehen deswegen oft im Abseits wirtschaftspolitischer Maßnahmen. Gleichwohl fällt es Verbrauchern offenkundig immer schwerer, mit den herkömmlichen Herausforderungen der Alltags- und Lebensökonomie zurecht zu kommen. Sie geraten in Gefahr, vom sozialen und wirtschaftlichen Leben ausgeschlossen zu werden, sei es durch Überschuldung, Krankheit oder mangelnde Kommunikationsmöglichkeiten. Dazu zählt auch das wachsende Problem der „Versorgungsarmut“, etwa durch hohe Energiekosten (vgl. Strünck 2011).

Merkmal des vertrauenden Verbrauchers ist, dass er sich auf die Politik und die anderen Marktakteure verlassen möchte und verlässt. Vermutlich aufgrund der mit dieser Grundhaltung einhergehenden Effizienz verhalten sich wohl die meisten Verbraucher wie ,vertrauende Verbraucher“. Sie wollen und können sich für eine Konsumentscheidung nicht zu viel Zeit nehmen und verwenden daher Vertrauen als einen Mechanismus der Komplexitätsreduktion (vgl. Kenning/Wobker 2012a, 2012b). Auch eine verbesserte Verbraucherbildung und -information kann daher nur bedingt dafür sorgen, dass sich alle Verbraucher ausreichend informieren, Kompetenz aneignen und die nötige Zeit investieren. Es sind vor allem die bereits genannten empirischen Forschungsergebnisse der Verhaltensökonomik, die auf diese Grenzen aufmerksam machen.

Eine dritte Gruppe von Konsumenten lässt sich schließlich als verantwortungsvolle Verbraucher beschreiben. Damit ist vor allem das Selbstverständnis dieser Gruppe gemeint. Das bereits beschriebene Phänomen des politischen Konsums findet man in dieser Gruppe vermutlich am ehesten. Zu unterscheiden sind dabei Verantwortung für sich selbst, für die Umwelt und für andere. Die Verantwortung bezieht sich auf die Produktebene von der Beschaffung im Markt über Ge- und Verbrauch bis hin zur Entsorgung. Verantwortungsvolle Verbraucher fühlen sich demzufolge verpflichtet, relevante Informationen einzuholen, auch wenn die Kapazitäten dafür begrenzt sind.

Auch wenn diese Einteilung nach Konsumfeldern, Situationen und Verbrauchertypen sehr unterschiedlich ausgeprägt sein kann, so kann sie doch einen ersten Beitrag dazu leisten, die Diskussion und Entwicklung auf der Ebene des Verbraucherleitbilds weiter zu differenzieren und voran zu treiben. Bis dato unklar geblieben ist im wissenschaftlichen Schrifttum, welche empirische Verbreitung die jeweiligen Typen haben. Die „Prävalenz" der verschiedenen Typen erscheint aber verbraucherwissenschaftlich und wirtschaftspolitisch überaus bedeutsam zu sein (vgl. Hagen 2011).

Vor diesem Hintergrund sollen im Folgenden die Konzeption, Methodik und die ersten Ergebnisse einer empirischen Studie vorgestellt werden, die zum Ziel hatte, das vom jeweiligen Verbraucher erworbene und verfügbare ökonomische Wissen zur Differenzierung und Quantifizierung der genannten Typen zu verwenden. Es soll an

trauend), vom „vulnerable consumer“ (verletzlich) und vom „responsible consumer“ (verantwortungsvoll) (vgl. Micklitz et al. 2010). 
dieser Stelle betont werden, dass diese Ergebnisse nur einen ersten Beitrag zu einer fortzuführenden Diskussion um das Verbraucherleitbild leisten können und sollen. Darüber hinaus bieten neuere empirische Studien, wie das unlängst erstmalig vom BMELV in Auftrag gegebene Gutachten zur Lage der Verbraucherinnen und Verbraucher in Deutschland, Hinweise auf die Verhaltensweisen der geschilderten Typen.

\section{Empirische Analyse zum Verbraucherwissen: Gibt es wirklich den mün- digen, wohl informierten Verbraucher?}

\subsection{Konzeption des typenbildenden Kriteriums}

Um einen empirisch informierten Beitrag zur Verbraucherleitbilddiskussion leisten und erste Hinweise auf die Ausprägung der nach ihrem ökonomischen Wissen differenzierten Verbrauchertypen zu erhalten (vgl. Lusardi/Mitchell 2011), wurde von den Autoren eine mehrstufige empirische Studie entwickelt. Da es in der Literatur nach wie vor an beschreibenden Merkmalen zur direkten Erfassung und Typologisierung fehlt, wurde in dieser Studie das von Wobker et al. (2012) konzipierte minimale ökonomische Wissen der Verbraucher als typenbildendes Kriterium verwendet. Dieses wurde analog zu Wobker et al. (2012: 5) definiert als das individuelle "basic knowledge of economic principles needed for understanding and participating in the economy". Konzeptionell wurde dabei über verschiedene Märkte hinweg das individuelle Verbraucherwissen über ökonomische Zusammenhänge, Fakten und Konzepte differenziert abgefragt. Die Fokussierung auf das minimale ökonomische Wissen der Verbraucher als typenbildendes Kriterium erschien aus den folgenden Gründen zweckmäßig:

(1) Aufbauend auf den Publikationen von Steiner (2001) und van Witteloostuijn (1990) kann davon ausgegangen werden, dass das ökonomische Wissen eines Verbrauchers das Ergebnis vergangener Erfahrungen und Lernprozesse ist und seine Problemlösungsfähigkeiten bestimmt. Insofern sollte sich im Wissen reflektieren, ob ein Verbraucher sich primär verantwortungsvoll oder vertrauend verhält, oder ein verletzlicher Verbraucher ist, der nur bedingt in der Lage ist, die grundlegenden ökonomischen Prinzipien und Regeln zu kennen, zu verstehen, zu erinnern und anzuwenden. Ist man bereit zu akzeptieren, dass das Verhalten der Vergangenheit zumindest teilweise einen Beitrag zur Erklärung künftigen Verbraucherverhaltens leisten kann, so kann das ökonomische Wissen auch als Indikator künftiger Verhaltensweisen und Problemlösungsfähigkeiten verwendet werden, die ja nach Micklitz et al. (2010) ein zentrales Unterscheidungsmerkmal der drei Typen darstellen.

(2) Eine wesentliche Facette im Kontext der Leitbilddiskussion ist die Verbraucherbildung (vgl. Oehler 2013). Ziel der Verbraucherbildung ist primär die Vermittlung von Verbraucherwissen. Die Idee dabei ist, dass durch die Vermittlung von Verbraucherwissen der Verbraucher in die Lage versetzt wird, „mündig“ bzw. „verantwortungsvoll“ und „souverän“ zu agieren. Insofern liegt der Unterteilung in die drei Gruppen oft als Metafaktor das Verbraucherwissen zugrunde, welches überwiegend ökonomisch interpretiert wird (vgl. zum Beispiel zur financial literacy: Lusardi/Mitchell 2011). 
(3) In der Wirtschafts- und Verbraucherpolitik herrscht, wie bereits beschrieben, nach wie vor ein Informationsparadigma vor (vgl. Kenning/Reisch 2013; Strünck 2011). Die Idee dabei ist, dass der Verbraucher zu wenige Informationen hat, um vernünftig bzw. mündig zu entscheiden, mithin schlecht informiert ist. Das Resultat der Informationsübertragung ist dabei regelmäßig der Wissenserwerb, ihr Ziel ist die Wissensmehrung oder, wie Strünck (2011: 3) pointiert formuliert, „Informationslücken zu stopfen“. Auch an dieser Stelle wird somit die hohe Bedeutung des minimalen ökonomischen Wissens für die erwähnte Typenbildung deutlich.

Vor diesem Hintergrund erscheint eine Differenzierung und Quantifizierung der drei genannten Typen mit Hilfe des Kriteriums „minimales ökonomischen Wissen“ (minimal economic knowledge - MEK) zweckmäßig. Konkret wurde hierzu die von Wobker et al. (2012) entwickelte Methodik zur Messung der individuellen MEKWerte verwendet, die das minimale Wirtschaftswissen in strukturierter Form in verschiedenen Bedarfsfeldern (z.B. Ernährung, Finanzen) erfasst. Im Hinblick auf die Details, die dieser Methodik zugrunde liegen, sei auf die entsprechende Originalquelle verwiesen.

\subsection{Stichprobe}

Um erste Hinweise auf die Verteilung des minimalen ökonomischen Wissens und die Merkmale der verschiedenen Verbrauchertypen zu erlangen, wurden mit Hilfe eines standardisierten Fragebogens 1.314 zufällig ausgewählte Personen in Deutschland mit Hilfe von CATI-Interviews befragt. ${ }^{4}$ Die Befragung erfolgte durch geschulte Interviewer und wurde von einem auf die Befragung von Verbrauchern spezialisiertem Marktund Meinungsforschungsunternehmen durchgeführt. Die Daten wurden im September 2010 erhoben. Die Stichprobe war durch folgende Merkmale gekennzeichnet (vgl. Tab. 1):

\begin{tabular}{llll}
\hline Geschlecht & weiblich $=50,5 \%$, & männlich $=49,5 \%$ & \\
\hline Alter & $\begin{array}{l}\text { Mittelwert } \\
=47 \text { Jahre }\end{array}$ & $\begin{array}{l}\text { Standard- } \\
\text { abweichung }=15\end{array}$ & $\begin{array}{l}\text { Range }= \\
\text { 12-85 Jahre }\end{array}$ \\
\hline Bildung & $\begin{array}{l}30,6 \% \text { mit Hoch- } \\
\text { schulabschluss }\end{array}$ & $\begin{array}{l}\text { 69,4\% ohne Hoch- } \\
\text { schulabschluss }\end{array}$ & \\
\hline $\begin{array}{l}\text { Durchschn. monatl. } \\
\text { HH-Netto-EK }\end{array}$ & Mittelwert & $\begin{array}{l}\text { Standard- } \\
\text { abweichung }=1.522 €\end{array}$ & $\begin{array}{l}\text { Range }= \\
0-10.000 €\end{array}$ \\
\hline
\end{tabular}

Tabelle 1: Merkmale der Stichprobe (Quelle: eigene Darstellung)

$4 \quad$ Zu den Details des Indikators vgl. Wobker et al. (2012). 


\subsection{Deskriptive Ergebnisse}

Zur Zuordnung eines Verbrauchers in eine der drei genannten Gruppen wurde entsprechend der Ausführungen im Kapitel 3.1. dessen individueller MEK-Wert verwendet. Dieser Wert wurde analog zu Wobker et al. (2012) wie folgt berechnet: Für jede richtige (falsche) Antwort erhielt der Proband einen (keinen) Punkt. Bei insgesamt 24 Fragen mit 0 oder 1 Punkten * (100/24) ergab sich ein minimaler Wert von 0 Punkten und ein maximaler Punktwert von 100. Für die gesamte Stichprobe ergab sich ein MEK-Mittelwert von 61,23 Punkten bei einer Standardabweichung von 14,8 Punkten und einer Range von 4,17-100,00 Punkten. Aufbauend auf den so ermittelten Werten wurden aus den Daten der gesamten Stichprobe drei Terzile gebildet (0-33, 34-66, 67-100). Dadurch wurden drei nicht gleich große Gruppen wie folgt identifiziert:

\section{Terzil 1 (0-33 Punkte): Die verletzlichen Verbraucher $(N=70)$}

Der Gruppe der verletzlichen Verbraucher wurden die Probanden zugeordnet, deren MEK-Wert zwischen 0 und 33 Punkten betrug. Insgesamt bestand diese Gruppe aus 70 Personen. Dies entsprach 5,3 Prozent der gesamten Stichprobe. Der MEKMittelwert in dieser Gruppe betrug 28,33 Punkte ( $\mathrm{SD}=6,04$; Range: $0-33,33)$. In dieser Gruppe waren 68,6 Prozent weiblich, 31,4 Prozent männlich. Das Durchschnittsalter betrug 39,77 Jahre (SD=15,78). 5,7 Prozent der Personen in dieser Gruppe hatten einen Hochschulabschluss. Das Haushalts-Netto-Einkommen in dieser Gruppe betrug 1.447 Euro ( $\mathrm{SD}=890$ Euro). Interessanterweise lag die Selbsteinschätzung hinsichtlich des eigenen Wissens relativ hoch $(\mathrm{MW}=2,43 ; \mathrm{SD}=0,91)$. Für den Wissenserwerb verwendet dieser Typ im Durchschnitt 2,56 Quellen ( $\mathrm{SD}=1,39)$.

\section{Terzil 2 (34-66 Punkte): Die vertrauenden Verbraucher $(N=826)$}

Die größte Prävalenz hatte die Gruppe der vertrauenden Verbraucher. Diesem Typus konnten 826 Personen der Gesamtstichprobe zugerechnet werden. Damit fielen 62,86 Prozent der Befragten in diese Gruppe. Der MEK-Mittelwert in dieser Gruppe betrug 55,93 Punkte (SD=8,76; Range: 33,34-66,67). Der Anteil der Männer war in dieser Gruppe größer als in der Gruppe der verletzlichen Verbraucher (57,5 Prozent weiblich, 42,5 Prozent männlich). Dies war zu erwarten, da bekannt ist, dass Männer oftmals vertrauensvoller sind als Frauen (vgl. Glaeser et al. 2000). Auch waren die vertrauenden Verbraucher etwas älter als die verletzlichen (MW=45,87 Jahre; $\mathrm{SD}=15,92$ Jahre). Der Anteil derjenigen mit Hochschulabschluss lag bei 23,8 Prozent; das mittlere Haushalts-Netto-Einkommen war deutlich höher als im ersten Terzil und betrug 2.155 Euro (SD=1469 Euro). Die Selbsteinschätzung lag bei einem Mittelwert von 3,06 $(\mathrm{SD}=0,81)$. Darüber hinaus verwendete dieser Typ mehr Quellen für den Wissenserwerb als der Verletzliche (MW=3,16; $\mathrm{SD}=1,85)$.

\section{Terzil 3 (67-100 Punkte): Die verantwortungsvollen Verbraucher $(\mathrm{N}=418)$}

Die zweitgrößte Gruppe in der von uns erhobenen Stichprobe waren die verantwortungsvollen Verbraucher (31,81 Prozent). Diese Gruppe erzielte einen durchschnittlichen MEK-Wert von 77,19 Punkten (SD=2,09; Range: 66,68-100). In dieser Gruppe überwogen die Männer (33,7 Prozent weiblich, 66,3 Prozent männlich). Darüber hinaus hat diese Gruppe das höchste Durchschnittsalter ( $M W=49,24$ Jahre; SD=15,27 Jahre). Zudem ist der Anteil derjenigen Befragten mit Hochschulabschluss in dieser 
Gruppe am höchsten (48,2 Prozent), ebenso das Haushalts-Netto-Einkommen: 2.642 Euro (SD=1647 Euro). Die Selbsteinschätzung betrug im Mittelwert 3,77 (SD=0,75). Die von diesem Typ verwendete Anzahl der Quellen für den Wissenserwerb lag bei 3,41 $(\mathrm{SD}=2,09)$ und damit erwartungsgemäß über derjenigen der beiden anderen Typen.

Insgesamt zeigt sich damit, dass der Typus des verletzlichen Verbrauchers in Deutschland die geringste Prävalenz hat. Gleichzeitig schätzt sich dieser Typ aber hinsichtlich seines ökonomischen Wissens insofern falsch ein, als dass er sein Wissen deutlich überschätzt. Am häufigsten findet man den ,vertrauenden“ Verbraucher. Darüber hinaus lässt sich etwa jeder dritte Verbraucher dem Typus „,verantwortungsvoll“ zuordnen. Insgesamt zeigen unsere Daten aber deutlich die bereits in der obigen Diskussion eingeführte Heterogenität der Verbraucher. Den Verbraucher gibt es somit nicht. Insofern bestätigt unsere Studie die Vermutung, dass eine undifferenzierte Leitbilddiskussion dem tatsächlichen Verbraucherverhalten nicht gerecht wird. Und auch wenn unsere Studie keine situativen Aspekte und Einflüsse erfassen wollte, so zeigt sie doch deutlich, dass es verschiedene Verbrauchertypen mit sehr unterschiedlichen Wissensständen, Kenntnissen und Problemlösungsfähigkeiten und nicht nur den vermeintlich mündigen Verbraucher gibt.

Aus diesen Ergebnissen lassen sich verschiedene Implikationen für die Verbraucherund damit verbunden die Wirtschaftspolitik ableiten. Da mit Blick auf den hier zur Verfügung stehenden Rahmen eine ausführliche Würdigung wenig zweckmäßig erscheint, sollen diese Implikationen an einem Beispiel, quasi pars pro toto angedeutet werden. Zur Veranschaulichung verwenden wir das für die Wirtschaftspolitik an vielen Stellen bedeutsame Wettbewerbsrecht und fokussieren in diesem Feld das Gesetz gegen Wettbewerbsbeschränkungen (GWB). Diese Fokussierung erscheint nicht nur aus Gründen der Übersichtlichkeit sinnvoll, sondern auch, weil gerade zwischen der Wettbewerbs- und der Verbraucherpolitik enge und unter Umständen konfliktgeladene Bezüge bestehen. So stellte beispielsweise Scherhorn (1975: 122) schon früh das Spannungsverhältnis dieser beiden Felder der Wirtschaftspolitik wie folgt heraus:

„(...) Verbraucherpolitik und Wettbewerbspolitik haben insofern unterschiedliche Zielsetzungen, als die erste darauf aus ist, dem Verbraucherinteresse Geltung zu verschaffen, während die zweite den Wettbewerb der Anbieter fördern will.“

Insofern ist der Transfer verbraucherwissenschaftlicher Erkenntnisse in die wirtschafts- und wettbewerbspolitische Diskussion im Sinne einer integrierten, widerspruchsfreien und effizienten Wirtschaftspolitik genauso wichtig wie naheliegend.

\section{Implikationen eines differenzierten Verbraucherleitbilds für die Wettbe- werbspolitik dargestellt am Beispiel des Verbraucherbegriffs des $\ 2$ GWB}

Im Gegensatz zum weitgehend uneinheitlichen Stand der Leitbilddiskussion in der Verbraucherpolitik ist die Wettbewerbspolitik überwiegend noch neoklassisch und informationsökonomisch geprägt. ${ }^{5}$ Während diese Prägung in weiten Teilen, insbesondere im makroökonomischen Kontext, unproblematisch und zielführend sein mag, 
führen diese unterschiedlichen Paradigmen aber immer dann zu Problemen, wenn verbraucher- und wettbewerbspolitische Aspekte miteinander in Berührung kommen. Besonders evident werden diese Probleme dann, wenn im wettbewerbspolitischen bzw. -rechtlichen Kontext die Figur des „Verbrauchers“ explizit Verwendung findet. Ein Beispiel hierfür ist der $\ 2$ Absatz 1 GWB („Freigestellte Vereinbarungen“). Dieser regelt die Ausnahmetatbestände des wettbewerbspolitisch und -rechtlich bedeutsamen $\ 1$ GWB und besagt im Wortlaut:

„(1) Vom Verbot des $\ 1$ freigestellt sind Vereinbarungen zwischen Unternehmen, Beschlüsse von Unternehmensvereinigungen oder aufeinander abgestimmte Verhaltensweisen, die unter angemessener Beteiligung der Verbraucher an dem entstehenden Gewinn zur Verbesserung der Warenerzeugung der -verteilung oder zur Förderung des technischen oder wirtschaftlichen Fortschritts beitragen, ohne dass den beteiligten Unternehmen: 1. Beschränkungen auferlegt werden, die für die Verwirklichung dieser Ziele nicht unerlässlich sind, oder 2. Möglichkeiten eröffnet werden, für einen wesentlichen Teil der betreffenden Waren den Wettbewerb auszuschalten."

Von besonderer Bedeutung für die Legitimation von Kooperation im Wettbewerb ist daher die Tatsache, dass „der Verbraucher an dem entstehenden Gewinn“ angemessen beteiligt wird. Der Nachweis dafür erscheint aber bereits auf den ersten Blick nahezu unmöglich, da die wirtschaftswissenschaftliche Analyse und darauf aufbauend, die empirische Studie gezeigt haben, dass es „den Verbraucher“ gar nicht gibt bzw. nicht geben kann. Es kann insofern nur darum gehen, dass ein Teil der Verbraucher oder aber alle Verbrauchertypen an dem entstehenden Gewinn beteiligt wird bzw. werden. Dabei bleibt aber vollkommen unklar, wie etwaige wettbewerbspolitisch bedingte Verteilungskonflikte zwischen den Verbrauchern und damit verbundene Benachteiligungen bestimmter Verbrauchertypen erkannt, bewertet und aufgelöst werden sollen.

Diese, dem undifferenzierten Verbraucherbegriff im $\ 2$ GWB innewohnende Problematik zeigt sich derzeit besonders deutlich in der kartellrechtlich geprägten Diskussion um das Verbot der vertikalen Preisbindung (vgl. Ahlert/Schefer 2012). Dieses Verbot wird aus dem $\ 1$ GWB i.V.m. Art. 101 Abs. 3 AEUV abgeleitet. In dieser Diskussion wird ausgehend vom Leitbild des mündigen Verbrauchers eine vertikale Preisbindung per se untersagt, obwohl nicht ausgeschlossen werden kann, dass diese zu Lasten bestimmter Dienstleistungsangebote geht, die gerade von den Verbraucher nachgefragt werden, die dem Typus des verletzlichen Verbrauchers zugerechnet werden können (vgl. Groehn/Kenning 2012). Insofern besteht die Gefahr, dass durch die Verengung des Leitbilds Effizienzgewinne im Segment des verantwortungsvollen Verbrauchers mit Effizienzverlusten im Segment des verletzlichen bzw. des vertrauenden Verbrauchers einhergehen und damit eine latente Umverteilung zwischen diesen beiden Gruppen stattfindet (vgl. hierzu weiterführend Haucap/Klein 2012; sowie Kenning/Wobker 2012a, 2012 b). So ist zum Beispiel bekannt, dass etwa 20 Prozent der Verbraucher den Preis als Indikator für Qualität verwenden und sich vor dem Hintergrund einer zunehmenden Verunsicherung hinsichtlich der auf den Märkten angebotenen Produkte (z.B. Lebensmittel) eine konstante Preispolitik der Anbieter wünschen (vgl. Kenning 2011: 178, 179). Es gibt also bei einem Teil der Verbraucher offensichtlich einen Bedarf an einer vertikalen Preiskoordination und -pflege, der bei 
einer undifferenzierten Auslegung des $₫ 2$ GWB angebotsseitig aber nicht gewährleistet werden kann. Unabhängig davon, wie diese Benachteiligung bestimmter PreisLeistungs-Kombinationen gerechtfertigt werden kann, führen diese Beschränkungen zu einer Reduktion von Vielfalt, was dem eigentlichen Gedanken der Wettbewerbspolitik unterläuft (vgl. hierzu Ahlert et al. 2011). Darüber hinaus zeigen aktuelle Studien im verbraucherpolitischen Kontext, wie z.B. das unlängst veröffentlichte erstmalige Gutachten zur Lage der Verbraucher, dass zahlreiche Faktoren einen Einfluss auf die Lage der Verbraucher haben können. Von besonderer Bedeutung scheint dabei das Konzept der Lebenslagen zu sein. Durch dessen situative Anlage entsteht im Kontext des $₫ 2$ GWB ein weiteres Problem, was sich u.a. darin manifestiert, dass bei der Beurteilung „entstehender Gewinne“ situative Aspekte eine Rolle spielen, die sich qua defintionem permanent (z.B. durch kundenseitiges Lernen) ändern können. Vor diesem Hintergrund stellt sich die Frage, wie sich diese grundsätzlichen und temporären Konflikte zwischen dem (impliziten) undifferenziertem wettbewerbspolitischen und dem uneinheitlichen und ausdifferenzierten verbraucherpolitischen Leitbild auf Dauer lösen lassen. Ein interessanter Ansatz könnte darin bestehen, die in der verbraucherpolitischen Diskussion begonnene Ausdifferenzierung wettbewerbspolitisch und rechtlich aufzugreifen und den von Ahlert und Schefer (2012) vorgeschlagenen Fallklassenansatz in diese Richtung weiter zu entwickeln. Dabei wären dann auch verhaltensbezogene bzw. verbraucherwissenschaftliche Erkenntnisse und Methoden zu berücksichtigen, so dass insgesamt eine politikfeldübergreifende Integration gelingen könnte, die eine effiziente Wirtschaftspolitik für beide Marktseiten ermöglichen würde.

\section{Zusammenfassung}

Gegenstand des vorliegenden Beitrags war es, den aktuellen Diskussionsstand um "das" Verbraucherleitbild aus einer wirtschaftswissenschaftlichen und -politischen Perspektive zu beleuchten. Dazu wurden zunächst die verschiedenen Paradigmen der ökonomischen Forschung hinsichtlich ihrer Diskussionsrelevanz gewürdigt. Im Ergebnis zeigte sich ein Zustand, den man als „Flickenteppich“ bezeichnen könnte und der angesichts aktueller wissenschaftlicher Erkenntnisse, insbesondere aus dem Bereich der Verhaltens- und Neuroökonomik, wohl dringend erneuert werden sollte. Da diese Erneuerung nicht ohne empirische Fundierung im Sinne einer in den Verbraucherwissenschaften bereits vielfach geforderten Evidenzbasierung gelingen kann, wurden im dritten Kapitel, aufbauend auf der in der aktuellen verbraucherpolitischen Diskussion vorgeschlagenen Typologisierung, die ersten Ergebnisse einer empirischen Studie zum minimalen ökonomischen Wissen der Verbraucher vorgestellt. Die Fokussierung auf das minimale ökonomische Wissen folgte der Idee, dass dieses Wissen als Indikator für die Problemlösungsfähigkeit und das Problemlösungsverhalten der Verbraucher im ökonomischen Kontext verwendet werden kann. Im Ergebnis zeigt sich, dass es den mündigen und wohl informierten Verbraucher in der Realität nicht gibt, sondern vielmehr verschiedene Typen zu unterscheiden sind. Zwar bilden die dem Idealbild der neoklassischen ökonomischen Theorie wohl am nächsten kommenden „verantwortungsvollen“ Verbraucher eine große Gruppe (31,8 Prozent); die weitaus höchste Prävalenz weist mit mehr als 62 Prozent jedoch die Gruppe der „vertrauenden Verbraucher" auf. Die kleinste Gruppe bilden schließlich mit etwas mehr als 5 Prozent die verletzlichen Verbraucher, die von der Wirtschaftspolitik auf den ersten 
Blick oft übersehen werden und interessanterweise die eigenen Kenntnisse und Fähigkeiten deutlich überschätzen. Da diese empirischen Befunde im Sinne einer integrierten und effizienten Wirtschaftspolitik auch für andere wirtschaftspolitische Bereiche von Bedeutung sein dürften, wurde im Folgenden am Beispiel des $\ 2$ GWB sowie dem Verbot der vertikalen Preisbindung gezeigt, welche Implikationen eine differenzierten Betrachtung mit sich bringen könnte. Im Ergebnis zeigt sich, dass eine ausdifferenzierte, empirisch informierte Leitbilddiskussion wirtschaftspolitisch von hoher Relevanz ist. Es bleibt zu hoffen, dass der vorliegende Beitrag eine entsprechende Berücksichtigung in dieser Diskussion finden wird und so eine verbrauchergerechte Politik und Rechtsprechung auf ökonomischer Basis ermöglicht.

\section{Literaturverzeichnis}

Ablert, D./Kenning, P./Olbrich, R./Scbröder, H. (Hrsg.) (2011): Vielfalt durch Gestaltungsfreiheit im Wettbewerb, München: C. H. Beck.

Ablert, D./Schefer, B. (2012): Vertikale Preis- und Markenpflege auf dem Prüfstand der (De-) Regulierung. Stranguliert das Kartellrecht den Wettbewerb als Entdeckungsverfahren?, in: Ahlert, D./Kenning, P./Olbrich, R./Schröder, H. (Hrsg.): Vertikale Preis- und Markenpflege im Kreuzfeuer des Kartellrechts, Wiesbaden: Springer Gabler, 5-68.

Ariely, D./Gockel, G./Zybak, M. (2010): Denken hilft zwar, nützt aber nichts: Warum wir immer wieder unvernünftige Entscheidungen treffen, München: Th. Knaur.

BMELV (2012): Politikstrategie Foodlabelling. Berichte über Landwirtschaft, in: Zeitschrift für Agrarpolitik und Landwirtschaft, Jg. 90/Heft 1, 35-69.

Camerer, C./Loewenstein, G./Prelec, D. (2005): Neuroeconomics: How neuroscience can inform economics, in: Journal of Economic Literature, Vol. 43/No. 1, 9-64.

Deppe, M./Schwindt, W./Pieper, A./Kugel, H./Plassmann, H./Kenning, P./Deppe, K./Ringelstein, E. (2007): Anterior cingulate reflects susceptibility to framing during attractiveness evaluation, in: Neuroreport, Vol. 18/No. 11, 1119-1123.

Deutscher Bundestag (2013): Bericht des Bundeskartellamtes über seine Tätigkeit in den Jahren 2011/2012 sowie über die Lage und Entwicklung auf seinem Aufgabengebiet, Deutscher Bundestag Drucksache 17/13675.

Fama, E. F. (1970): Efficient Capital Markets: A Review of Theory and Empirical Work, in: Journal of Finance, Vol. 25/No. 2, 383-417.

FDP (2013). Bürgerprogramm 2013. Damit Deutschland stark bleibt.

Fritsch, M./Wein, T./Ewers, H.-J. (1998): Marktversagen und Wirtschaftspolitik, Mikroökonomische Grundlagen staatlichen Handelns, 3. Aufl., München: Vahlen.

Frey, B. (1990): Entscheidungsanomalien: Die Sicht der Ökonomie, in: Psychologische Rundschau, Jg. 41, April, 67-83.

Gigerenzer, G. (1991): From tools to theories: A heuristic of discovery in cognitive psychology, in: Psychological Review, Vol. 98/No. 2, 254-267.

Glaeser, E. L/Laibson, D. I./Scheinkman, J. A./Soutter, C. L. (2000): Measuring Trust, in: Quarterly Journal of Economics, Vol. 115/No. 3, 811-846.

Groebn, A./Kenning, P. (2012). Sollte das Verbot der vertikalen Preisbindung abgeschafft werden? Ein Plädoyer für mehr Freiheit im vertikalen Marketing. Absatzwirtschaft (asw), Heft 11, 32-35. 
Grugel, C. (2012). Verbraucherforschung wird wichtiger, Journal für Verbraucherschutz und Lebensmittelsicherheit, in: Journal of Consumer Protection and Food Safety, Vol. 7/No. 10, 101-103.

Hagen, K. (2011): Wirksame Beratung der Verbraucherpolitik setzt unabhängige Daten über das Verhalten von Verbrauchern voraus, in: Wochenbericht des DIW Berlin, Heft 25, $18-24$.

Hagen, K./Micklitz, H.-W./Oebler, A./Reisch, L.A./Strünck, C. (2013): Check Verbraucherpolitik und Verbraucherbeteiligung: Empfehlungen für eine evidenzbasierte Verbraucherpolitik, Journal für Verbraucherschutz und Lebensmittelsicherheit, Jg. 8/Heft 1-2, 61-66.

Haucap, J./Klein, G. (2012): Einschränkungen der Preisgestaltung im Einzelhandel aus wettbewerbsökonomischer Perspektive, in: Ahlert, D./Kenning, P./Olbrich, R./Schröder, H. (Hrsg.): Vertikale Preis- und Markenpflege im Kreuzfeuer des Kartellrechts, Wiesbaden: Springer Gabler, 169-186.

Hellmann, K.U. (2003): Soziologie der Marke, Frankfurt/M.: Suhrkamp.

Hirshleifer, J./Riley, J. G. (1979): The Analytics of Uncertainty and Information - An Expository Survey, in: Journal of Economic Literature, Vol. 17/No. 4, 1375-1421.

Kahneman, D. (2002): Maps of bounded rationality: A perspective on intuitive judgment and choice, Nobel Prize Lecture, Stockholm. Link: http://www.nobelprize.org/ nobel_prizes/economic-sciences/laureates/2002/kahne-mann-lecture.pdf (zuletzt abgerufen am 19.08. 2013).

Kenning, P. (2011): Kritische Würdigung der kartellrechtlichen Regulierung der vertikalen Preisund Markenpflege aus Verbrauchersicht - unter besonderer Berücksichtigung der Informationsökonomik und der Behavioral Economics, in: Ahlert, D./Kenning, P./Olbrich, R./Schröder, H. (Hrsg.): Vielfalt durch Gestaltungsfreiheit, München: C. H. Beck, 155-207.

Kenning, P./Linzmajer, M. (2011): Consumer Neuroscience - An Overview of an Emerging Discipline with Implications for Consumer Policy, in: Journal of Consumer Protection and Food Safety, Vol. 6/No. 1, 111-125.

Kenning, P./Plassmann, H. (2005): NeuroEconomics: An overview from an economic perspective, Brain Research Bulletin, Vol. 67/No. 15, 343-354.

Kenning, P./Plassmann, H./Ablert, D. (2007): Consumer Neuroscience - Implikationen neurowissenschaftlicher Forschung für das Marketing, in: Marketing - Zeitschrift für Forschung \& Praxis, Jg. 29/Heft 1, 7-68.

Kenning, P./Reisch, L. (2013): Alternativen zum Informationsparadigma der Verbraucherpolitik: Eine kommentierende Einführung in ein noch dynamisches verbraucherwissenschaftliches Feld mit verbraucherpolitischen Implikationen, in: Journal of Consumer Protection and Food Safety. Link: http://link.springer.com/content/pdf/10.1007\%2Fs00003 -013-0832-1.pdf (zuletzt abgerufen am 19.08. 2013).

Kenning, P./Wobker, I. (2012a): Die negativen Folgen von Kundenverwirrtheit und die moderierende Rolle von Vertrauen in der Konsumgüterdistribution: Konzeptionelles Modell und empirische Befunde, in: Ahlert, D./Kenning, P./Olbrich, R./Schröder, H. (Hrsg.): Vertikale Preis- und Markenpflege im Kreuzfeuer des Kartellrechts, Wiesbaden: Springer Gabler, 69-94.

Kenning P./Wobker, I. (2012b): Affektive und kognitive Verhaltensstrategien zur Überwindung von Informationsasymmetrien im Konsumgüterhandel - eine empirische Analyse mit kartellrechtlichen Implikationen, in: Betriebswirtschaftliche Forschung und Praxis, Jg. 64/Heft 6, 626-642.

Lusardi, A./Mitchell, O. S. (2011): Financial Literacy around the World: An Overview, in: Journal of Pension Economics and Finance, Vol. 10/No. 4, 497-508. 
Micklitz, H.-W./Oebler, A./Piorkowsky, M.-B./Reisch, L.A./Strünck, C. (2010): Der vertrauende, der verletzliche oder der verantwortungsvolle Verbraucher? Plädoyer für eine differenzierte Strategie in der Verbraucherpolitik. Stellungnahme des Wissenschaftlichen Beirats für Verbraucher- und Ernährungspolitik beim BMELV, Berlin.

Oebler, A. (2013): Neue alte Verbraucherleitbilder: Basis für die Verbraucherbildung? in: Haushalt in Bildung und Forschung, Jg. 2/Heft 2, 44-60.

Oebler, A./Reisch, L. A. (2008): Behavioural Economics - eine neue Grundlage für die Verbraucherpolitik? Eine Studie im Auftrag des vzbv e.V., Bamberg/Berlin/Kopenhagen: vzbv e.V.

Olbrich, R./Bubr, C.-C. (2007): Preisbindungsverbot und Wettbewerb auf dem Konsumgütersektor, in: Das Wirtschaftsstudium, Jg. 36/Heft 11, 1439-1445.

Piorkowsky, M.-B. (2004): Ökonomie des privaten Haushalts, in: May, H. (Hrsg.): Lexikon der ökonomischen Bildung, 5., aktualisierte und erweiterte Aufl., München, Wien: R. Oldenbourg Verlag, 382-384.

Priddat B. P. (2007): Neuroökonomie - Neue Theorie zu Konsum, Marketing und emotionalem Verhalten in der Ökonomie, Marburg: Metropolis-Verlag.

Scheibehenne, B./Greifeneder, R./Todd, P. M. (2010): Can There Ever Be Too Many Options? A Meta-Analytic Review of Choice Overload, in: The Journal of Consumer Research, Vol. 37/No. 3, 409-425.

Scherhorn, G. (1975): Verbraucherinteresse und Verbraucherpolitik, Göttingen: Schwartz-Verlag. Schneider, F. (2010): Geleitwort zu den Forschungsergebnissen des Projektes WENKE ${ }^{2}$, in: Antoni-Komar, I./Lehmann-Waffenschmidt, M./Pfriem, R./Welsch, H. (Hrsg.): $W_{E N K E}^{2}$ - Wege zum nachhaltigen Konsum, Marburg: Metropolis-Verlag, 9-11.

Schrader, U./Diebl, B. (2010): Nachhaltigkeitsmarketing durch Interaktion, in: Marketing Review St. Gallen, Vol. 27/No. 5, 16-20.

Srnka, K. J./Schweitzer, F. M. (2000): Macht, Verantwortung und Information: Der Konsument als Souverän?, in: Zeitschrift für Wirtschafts- und Unternehmensethik, Jg. 1/Heft 2, 192-205.

Steiner, P. (2001): The Sociology of Economic Knowledge, in: European Journal of Social Theory, Vol. 4/No. 4, 443-458.

Strünck, C. (2011): Die Verbraucherpolitik braucht Pragmatismus statt wirklichkeitsferner Leitbilder, in: Wirtschaftsdienst: Zeitschrift für Wirtschaftspolitik, Jg. 91/Heft 3, 165-168.

Strünck, C./Arens-Azevêdo, U./Brönneke, T./Hagen, K./Jaquemoth, M./Kenning, P./Liedtke, C./Oebler, A./Schrader, U./Tamm, M. (2012): Ist der „mündige Verbraucher“ ein Mythos? Auf dem Weg zu einer realistischen Verbraucherpolitik. Stellungnahme des Wissenschaftlichen Beirats Verbraucher- und Ernährungspolitik beim BMELV, Berlin.

Strünck, C./Becker, T./Jungermann, H./Leonbäuser, I./Micklit々, H.-W./Oebler, A./Piorkowsky, M.B./Reisch, L. A. (2010): Wollen wirklich alle den „mündigen Verbraucher“? Wie Interessengruppen ein Leitbild instrumentalisieren, Stellungnahme des Wissenschaftlichen Beirats Verbraucher- und Ernährungspolitik beim BMELV, Berlin. Link: http:// www.bmelv.de/SharedDocs/Downloads/Ministerium/Beiraete/Verbraucherpoltik/20 10_12_InteressengruppeMuendigerVerbraucher.pdf;jsessionid=D798E7D264FCE614 D2CDB6E1D2DD46E8.2_cid358?_blob=publicationFile (zuletzt abgerufen am 19.08.2013).

Sunstein, C. R. (2012): Impersonal Default Rules vs. Active Choices vs. Personalized Default Rules: A Triptych, Working Paper. Link: http://dash.harvard.edu/bitstream/ handle/1/9876090/Decidingbydefault11_5.pdf?sequence=1 (zuletzt abgerufen am 19.08.2013). 
Wobker, I./Lebmann-Waffenschmidt, M./Kenning, P./Gigerenzer, G. (2012): What do People Know About the Economy? A Test of Minimal Economic Knowledge in Germany, Dresden Discussion Paper Series in Economics, No. 3/12.

Weber, E. U./Johnson, E. J. (2009): Mindful Judgment and Decision Making, in: Annual Review of Psychology, Vol. 60/No. 53, 53-85.

Weiber, R./Adler, J. (1995a): Informationsökonomisch begründete Typologisierung von Kaufprozessen, in: Zeitschrift für betriebswirtschaftliche Forschung, Jg. 47/Heft 1, 43-65.

Weiber, R./Adler, J. (1995b): Positionierung von Kaufprozessen im informationsökonomischen Dreieck: Operationalisierung und verhaltenswissenschaftliche Prüfung, in: Zeitschrift für betriebswirtschaftliche Forschung, Jg. 47/Heft. 2, 99-123.

Witteloostuijn, A. van (1990): Leaning in Economic Theory: A Taxonomy with an Application to Expectations Formation, in: Journal of Economic Psychology, Vol. 11/No. 2, 183-207. 